\title{
Richardson Cascadic Multigrid Method for 2D Poisson Equation Based on a Fourth Order Compact Scheme
}

\author{
Li Ming ${ }^{1}$ and Li Chen-Liang ${ }^{2}$ \\ ${ }^{1}$ Department of Mathematics, Honghe University, Mengzi, Yunnan 661100, China \\ ${ }^{2}$ School of Mathematics and Computing Science, Guilin University of Electronics Technology, Guilin, Guangxi 541004, China \\ Correspondence should be addressed to Li Ming; mathlm@126.com
}

Received 9 September 2013; Revised 31 December 2013; Accepted 2 January 2014; Published 10 March 2014

Academic Editor: Song Cen

Copyright (C) 2014 L. Ming and L. Chen-Liang. This is an open access article distributed under the Creative Commons Attribution License, which permits unrestricted use, distribution, and reproduction in any medium, provided the original work is properly cited.

Based on a fourth order compact difference scheme, a Richardson cascadic multigrid (RCMG) method for 2D Poisson equation is proposed, in which the an initial value on the each grid level is given by the Richardson extrapolation technique (Wang and Zhang (2009)) and a cubic interpolation operator. The numerical experiments show that the new method is of higher accuracy and less computation time.

\section{Introduction}

Poisson equation is a partial differential equation (PDE) with broad applications in theoretical physics, mechanical engineering and other fields, such as groundwater flow $[1,2]$, fluid pressure prediction [3], electromagnetics [4], semiconductor modeling [5], and electrical power network modeling [6].

We consider the following two-dimensional (2D) Poisson equation:

$$
\begin{gathered}
-\frac{\partial^{2} u(x, y)}{\partial x^{2}}-\frac{\partial^{2} u(x, y)}{\partial y^{2}}=f(x, y), \quad \text { in } \Omega, \\
u(x, y)=0, \quad \text { on } \partial \Omega,
\end{gathered}
$$

where $\Omega \in R^{2}$ is a rectangular domain or union of rectangular domains with Dirichlet boundary $\partial \Omega$. The solution $u(x, y)$ and the forcing function $f(x, y)$ are assumed to be sufficiently smooth.

Multigrid (MG) method is one of the most effective algorithms to solve the large scale problem. In 1996, cascadic multigrid (CMG) method proposed by Bornemann and Deuflhard [7] and then analyzed by Shi et al. (see [8-11]) and Shaidurov (see [12]). In the recent years, there have been several theoretical analyses and the applications of these methods for the plate bending problems (see [13]), the parabolic problems (see [10]), the nonlinear problems (see $[14,15])$, and the Stokes problems (see [16]). In order to improve the efficiency of the CMG, some new extrapolation formulas and extrapolation cascadic multigrid (EXCMG) methods are proposed by Chen et al. (see [17-20]). These new methods can provide a better initial value for smoothing operator on the refined grid level to accelerate their convergence rate.

Based on the Richardson extrapolation technique, Wang and Zhang [21] presented a multiscale multigrid algorithm. Numerical experiments show that the new method is of higher accuracy solution and higher efficiency.

In this paper, in order to develop a more efficient CMG method, we use the Richardson extrapolation technique presented in [21] and a new extrapolation formula; a new Richardson extrapolation cascadic multigrid (RCMG) method for 2D Poisson equation is proposed.

The sections are arranged as follows: the fourth order compact difference scheme and Richardson extrapolation technique are given in Section 2. Chen's new extrapolation 
formula and EXCMG method are introduced in Section 3. In Section 4, we present the RCMG method. In Section 5, the numerical experiments show the effectiveness of the new method.

\section{Fourth Order Compact Difference Scheme and Richardson Extrapolation Technique}

For convenience, we consider the rectangular domain $\Omega=$ $\left[0, L_{x}\right] \times\left[0, L_{y}\right]$. We discretize $\Omega$ with uniform mesh sizes $h_{x}=L_{x} / N_{x}$ and $h_{y}=L_{y} / N_{y}$ in the $x$ and $y$ coordinate directions. The mesh points are $\left(x_{i}, y_{j}\right)$ with $x_{i}=i h_{x}$ and $y_{j}=j h_{y}$, and $0 \leq i \leq N_{x}, 0 \leq j \leq N_{y}$. Let's denote the mesh aspect ratio $\gamma=h_{x} / h_{y}$, and $u_{i, j}$ be the solution at the grid point $\left(x_{i}, y_{j}\right)$, we can rewrite the fourth order compact difference scheme of (1) into the following form [22]:

$$
\begin{aligned}
a u_{i j}+ & b\left(u_{i+1, j}+u_{i-1, j}\right)+c\left(u_{i, j+1}+u_{i, j-1}\right) \\
& +d\left(u_{i+1, j+1}+u_{i+1, j-1}+u_{i-1, j+1}+u_{i-1, j-1}\right) \\
= & \frac{h_{x}^{2}}{2}\left(8 f_{i, j}+f_{i+1, j}+f_{i-1, j}+f_{i, j}+f_{i, j-1}\right) .
\end{aligned}
$$

The coefficients in (2) are

$$
\begin{gathered}
a=-10\left(1+\gamma^{2}\right), \quad b=5-\gamma^{2}, \\
c=5 \gamma^{2}-1, \quad d=\frac{\left(1+\gamma^{2}\right)}{2} .
\end{gathered}
$$

If the domain $\Omega$ is subdivided into a sequence of grids $Z_{l h}$ (or $\left.Z_{l}\right), l=0,1,2, \ldots, L$ with step length $h_{l}=h / 2^{l}=h_{l, x}=h_{l, y}$ (namely, $\gamma=1$ ), by using the fourth order compact difference scheme (see (2)), a series of linear equations of the model problem (1) are given as follows

$$
A^{l} u^{l}=F^{l}, \quad l=0,1,2, \ldots, L .
$$

Assume the fourth order accurate solutions $u_{i, j}^{2 h}$ and $u_{i, j}^{h}$ on the $Z_{2 h}$ grid and the $Z_{h}$ grid are given, respectively (Figure 1). In 2009, Wang and Zhang [21] applied the Richardson extrapolation (where $p=4$ )

$$
\tilde{u}_{i, j}^{2 h}=\frac{\left(2^{p} u_{2 i, 2 j}^{h}-u_{i, j}^{2 h}\right)}{2^{p}-1}=\frac{\left(16 u_{2 i, 2 j}^{h}-u_{i, j}^{2 h}\right)}{15}
$$

to get a sixth order accurate solution $\tilde{u}_{i, j}^{2 h}$ on $Z_{2 h}$.

The above extrapolation operator is rewritten as the following iterative operator RET.

Algorithm 1. Consider $\widetilde{u}^{h \text {,new }} \leftarrow \operatorname{RET}\left(\widetilde{u}^{h}, \widetilde{u}^{2 h}, \varepsilon, k^{\max }\right)$.

Step 1. Set $\widetilde{u}^{h \text { old }}:=\widetilde{u}^{h}, k:=0$.

Step 2. Update every (even, even) grid point on $Z_{h}$ by Richardson extrapolation formula (see (5)); then use direct interpolation to get $\widetilde{u}_{2 i, 2 j}^{h, \text { new }} \in Z_{h}$. Consider

$$
\widetilde{u}_{2 i, 2 j}^{h, \text { new }}:=\frac{\left(16 u_{2 i, 2 j}^{h, \text { old }}-u_{i, j}^{2 h}\right)}{15} .
$$

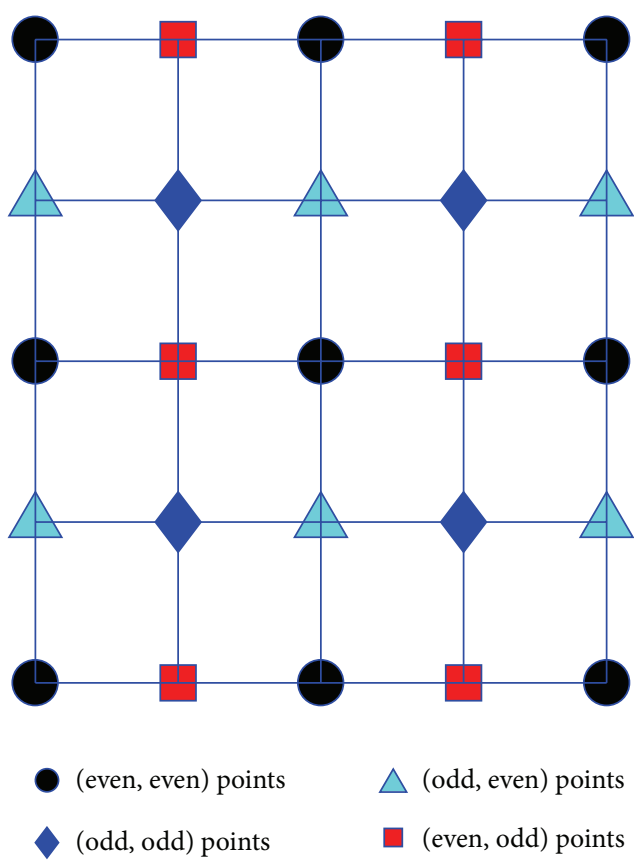

FIGURE 1: Four types of points on $4 \times 4$ grid.

Step 3. Update every (odd, odd) grid point on $Z_{h}$. From (2), for each (odd, odd) point $(i, j)$, the updated solution is

$$
\begin{aligned}
\tilde{u}_{i, j}^{h, \text { new }}:=\frac{1}{a}[ & F_{i, j}-b\left(\widetilde{u}_{i+1, j}^{h, \text { old }}+\widetilde{u}_{i-1, j}^{h \text { old }}\right) \\
& -c\left(\widetilde{u}_{i, j+1}^{h \text {,old }}+\widetilde{u}_{i, j-1}^{h, \text { old }}\right) \\
& \left.-d\left(\tilde{u}_{i+1, j+1}^{h \text {,new }}+\widetilde{u}_{i+1, j-1}^{h, \text { new }}+\widetilde{u}_{i-1, j+1}^{h \text {,new }}+\widetilde{u}_{i-1, j-1}^{h, \text { new }}\right)\right] .
\end{aligned}
$$

Here, $F_{i, j}$ represents the right-hand side part of (2).

Step 4. Update every (odd, even) grid point on $Z_{h}$. From (2), for each (odd, even) grid point, the updated value is

$$
\begin{aligned}
\widetilde{u}_{i, j}^{h, \text { new }}:=\frac{1}{a}[ & F_{i, j}-b\left(\widetilde{u}_{i+1, j}^{h, \text { new }}+\widetilde{u}_{i-1, j}^{h, \text { new }}\right) \\
& -c\left(\widetilde{u}_{i, j+1}^{h \text {,new }}+\widetilde{u}_{i, j-1}^{h, \text { new }}\right) \\
& \left.-d\left(\tilde{u}_{i+1, j+1}^{h \text { old }}+\tilde{u}_{i+1, j-1}^{h \text {,old }}+\tilde{u}_{i-1, j+1}^{h \text { old }}+\tilde{u}_{i-1, j-1}^{h, \text { old }}\right)\right] .
\end{aligned}
$$

Step 5. Update every (even, odd) grid point on $Z_{h}$. From (2), the idea is similar to the (odd, even) grid point. Let $k:=k+1$.

Step 6. If $\left\|\widetilde{u}^{h \text {,new }}-\widetilde{u}^{h \text {,old }}\right\| \leq \varepsilon$ or $k=k^{\text {max }}$, stop. Else, let $\widetilde{u}^{h \text { old }}:=\widetilde{u}^{h, \text { new }}$ and return to Step 3 .

\section{New Extrapolation Formula and EXCMG Method}

Based on an asymptotic expansion of finite element method, a new extrapolation formula and an extrapolation cascadic 
multigrid (EXCMG) method are proposed by Chen et al. (see [17-20]). The numerical experiments show that the EXCMG method is of high accuracy and efficiency. Now we rewrite the new extrapolation formula as follows.

$$
\begin{aligned}
& \operatorname{Ex} \tilde{u}_{2 i, 2 j}^{h}:=\frac{\left(5 u_{2 i, 2 j}^{h}-u_{i, j}^{2 h}\right)}{4}, \\
& \operatorname{Ex} \widetilde{u}_{2 i+1, j}^{h}:=u_{2 i+1, j}^{h}+\frac{\left[\left(u_{2 i, 2 j}^{h}-u_{i, j}^{2 h}\right)+\left(u_{2 i+2,2 j}^{h}-u_{i+1, j}^{2 h}\right)\right]}{8}, \\
& \operatorname{Ex}_{2 i, 2 j+1}^{h}:=u_{2 i, 2 j+1}^{h} \\
&+\frac{\left[\left(u_{2 i, 2 j}^{h}-u_{i, j}^{2 h}\right)+\left(u_{2 i, 2 j+2}^{h}-u_{i, j+1}^{2 h}\right)\right]}{8}, \\
& \operatorname{Ex}_{2 i+1,2 j+1}^{h}:=u_{2 i+1,2 j+1}^{h} \\
&+\left[\left(u_{2 i, 2 j}^{h}-u_{i, j}^{2 h}\right)+\left(u_{2 i+2,2 j}^{h}-u_{i+1, j}^{2 h}\right)\right. \\
&+\left(u_{2 i, 2 j+2}^{h}-u_{i, j+1}^{2 h}\right) \\
&\left.+\left(u_{2 i+2,2 j+2}^{h}-u_{i+1, j+1}^{2 h}\right)\right] \\
& \times
\end{aligned}
$$

Let us denote the above new extrapolation formula by operator

$$
\operatorname{Ex} \widetilde{u}^{h}:=F\left(u^{2 h}, u^{h}\right) \text {. }
$$

Now let $\bar{u}^{i}$, on $Z_{i}, i=0,1$ denote the exact solutions, the EXCMG method is as following:

Algorithm 2 (EXCMG). For $l=2, \ldots, L$, consider the following

Step 1. Extrapolate by using the new extrapolation formula (see (10))

$$
\operatorname{Ex} \widetilde{u}^{l-1}:=F\left(\bar{u}^{l-2}, \bar{u}^{l-1}\right) .
$$

Step 2. Compute the initial value

$$
u^{l, 0}:=I_{2} \operatorname{Ex} \tilde{u}^{l-1}
$$

on $Z_{l}$ by using quadratic interpolation operator $I_{2}$. Step 3. Smooth $m_{l}$ times to get the iterative solution

$$
\vec{u}^{l}:=S_{l}^{m_{l}} u^{l, 0}
$$

on $Z_{l}$ by using some classical iterative operator $S_{l}$.

Step 4. Return to Step 1 if $l<L$, until you get the final iterative solution $\bar{u}^{L}$ on the finest grid $Z_{L}$.

\section{Richardson Cascadic Multigrid Method}

One of the main tasks in cascadic multigrid method is constructing a suitable interpolation. Based on a new extrapolation-interpolation formula, Chen [17-20] proposed the following extrapolation cascadic multigrid (EXCMG) method, in which the new extrapolation and quadratic interpolation are used to provide a better initial value on refined grid.

In this section, we use RET operator and a cubic interpolation to interpolate the initial guess $\widetilde{u}^{l, 0}$ on the refined grid $Z_{l h}$. Then a classical iterative operator (such as conjugate gradient method) is used as a smoothing operator to compute the high accuracy solution on the fine grid $Z_{l h}$. Similar to the standard CMG method, we propose the following Richardson cascadic multigrid (RCMG) method.

Algorithm 3 (RCMG).

Step 1. Exactly solve the equation $A^{l} u^{l}=F^{l}$ on coarsest grid $Z_{l}, l=1,2$.

Step 2. Run Algorithm 1; we have

$$
\bar{u}^{l}=\operatorname{RET}\left(u^{l}, u^{l-1}, \varepsilon, k_{l}^{\max }\right) .
$$

Step 3. Use a cubic interpolation operator $I_{3}$ to have the initial value

$$
u^{l+1,0}:=I_{3} \bar{u}^{l}
$$

on the gird level $Z_{l+1}$.

Step 4. Smoothing $w_{l}$ times by using the classical iterative operator $S_{l}$,

$$
u^{l+1}:=S_{l}^{w_{l}} u^{l+l, 0}
$$

on the level $Z_{l+1}$. Set $l:=l+1$;

Step 5. Return to Step 2, if $l<L$.

The difference between RCMG method and EXCMG method is that

$$
\begin{aligned}
\mathrm{RCMG}= & \mathrm{RET}+\text { cubic interpolation } \\
& + \text { classical iterative operator }+\mathrm{CMG} \\
\mathrm{EXCMG}= & \text { new extrapolation }+ \text { quadratic interpolation } \\
& + \text { classical iterative operator }+\mathrm{CMG} .
\end{aligned}
$$

\section{Numerical Experiment and Comparison}

Numerical experiments are conducted to solve a 2D Poisson equation $(1)$ on the unit square domain $[0,1] \times[0,1]$. 


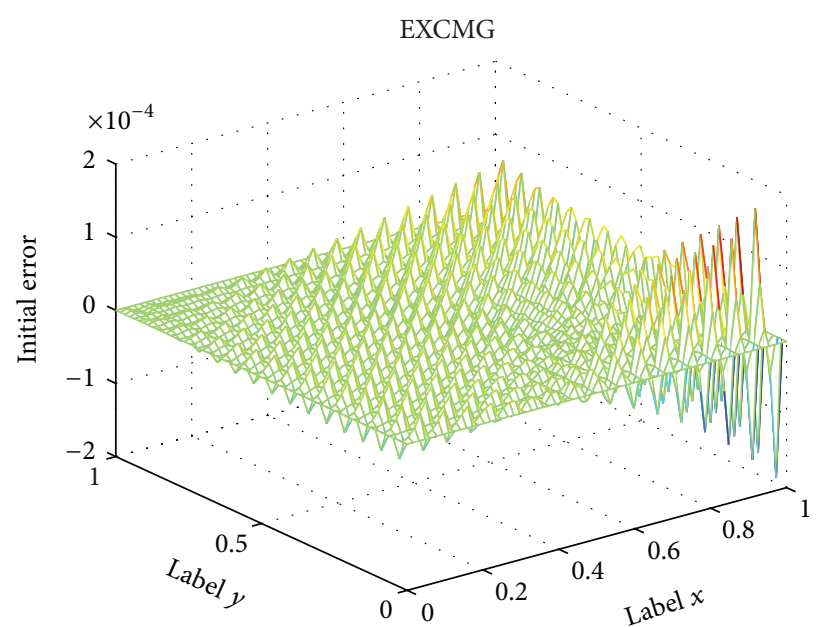

(a)

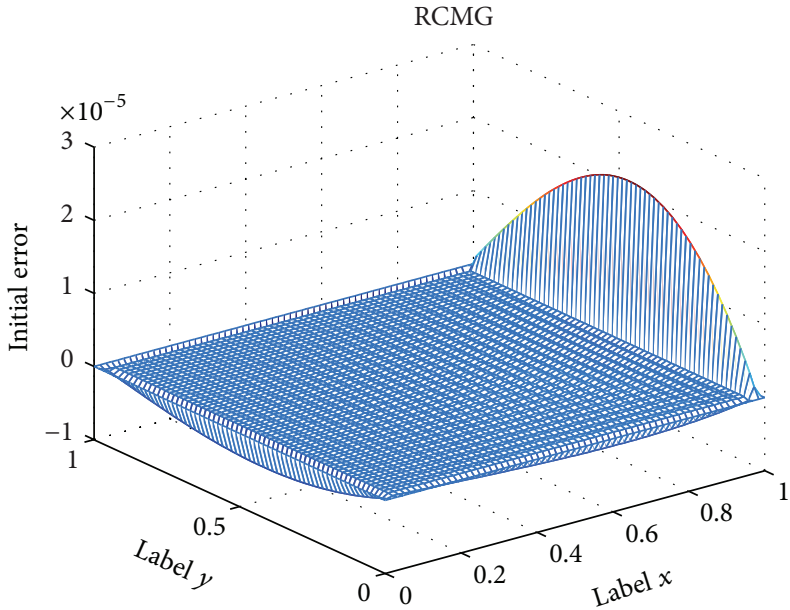

(b)

FIgURE 2: Example 4, grid $64 \times 64$, initial error of EXCMG ((a) scale $\left.10^{-4}\right)$ and RCMG ((b) scale $10^{-5}$ ).

Example 4. The exact solution $u=\sin (y)\left(1-e^{x}\right)\left(1-x^{2}\right)(1-$ $\left.y^{2}\right)$; the forcing function

$$
\begin{aligned}
f= & 2 \sin (y)\left(e^{x}-1\right)\left(x^{2}-1\right)+2 \sin (y)\left(e^{x}-1\right)\left(y^{2}-1\right) \\
& -\sin (y)\left(e^{x}-1\right)\left(x^{2}-1\right)\left(y^{2}-1\right) \\
& +4 y \cos (y)\left(e^{x}-1\right)\left(x^{2}-1\right) \\
& +4 x e^{x} \sin (y)\left(y^{2}-1\right)+e^{x} \sin (y)\left(x^{2}-1\right)\left(y^{2}-1\right) .
\end{aligned}
$$

Example 5. The exact solution $u=\ln (1+$ $\left.\sin \left(\pi x^{2}\right)\right)(\cos (\sin (x))-1) \sin (\pi y)$; the forcing function

$$
\begin{aligned}
f= & \pi^{2} \sin (\pi y) \log \left(\sin \left(\pi x^{2}\right)+1\right)(\cos (\sin (x))-1) \\
& -\sin (\sin (x)) \sin (\pi y) \log \left(\sin \left(\pi x^{2}\right)+1\right) \sin (x) \\
& +\cos (\sin (x)) \sin (\pi y) \log \left(\sin \left(\pi x^{2}\right)+1\right) \cos ^{2}(x) \\
& -\frac{2 \pi \sin (\pi y) \cos \left(\pi x^{2}\right)(\cos (\sin (x))-1)}{\sin \left(\pi x^{2}\right)+1} \\
& +\frac{\left(4 \pi^{2} x^{2} \sin (\pi y) \cos ^{2}\left(\pi x^{2}\right)(\cos (\sin (x))-1)\right)}{\left(\sin \left(\pi x^{2}\right)+1\right)^{2}} \\
& +\frac{4 \pi^{2} x^{2} \sin (\pi y) \sin \left(\pi x^{2}\right)(\cos (\sin (x))-1)}{\sin \left(\pi x^{2}\right)+1} \\
& +\frac{4 \pi x \sin (\sin (x)) \sin (\pi y) \cos (x) \cos \left(\pi x^{2}\right)}{\sin \left(\pi x^{2}\right)+1} .
\end{aligned}
$$

We use the conjugate gradient (CG) method as a smoothing iterative operator $S$ in EXCMG method and RCMG

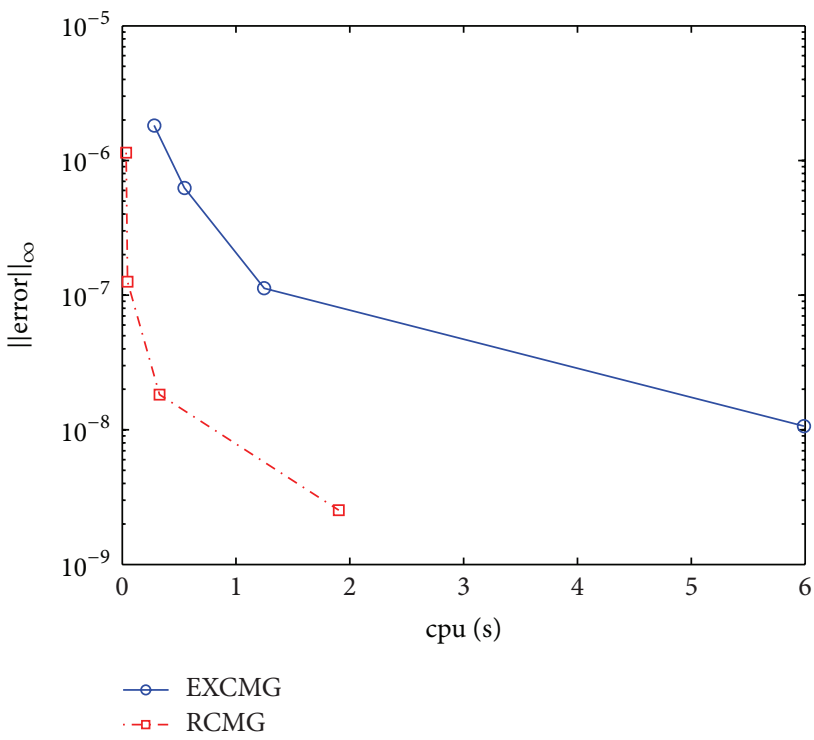

Figure 3: Comparison of the maximum error $\left\|\bar{u}^{L}-u\right\|_{\infty}$ and cpu time for Example 4 with $L=3$, taking step lengths $h_{L}=1 / m, m=$ $64,128,256$, and 512 , respectively.

method. In EXCMG method, the number of iterations $\widehat{m}_{l}$ on each grid level has to increase from finer to coarser grids; in this paper let $\widehat{m}_{l}=8 \times 2^{L-l+1}$. And in RCMG, we set the number of iteration $k_{l}^{\max }$ (Step 2) and $w_{l}$ (Step 4) be $8 \times 2^{L-l}$. We set $\varepsilon=10^{-8}$ of RET in the RCMG method (on Step 2).

5.1. Comparison of the Initial Errors. Assume that the exact solutions of the difference equation on grids $16 \times 16$ and $32 \times 32$ are given. We compare EXCMG method with RCMG method for the initial error $\left\|\operatorname{Err}_{64}^{0}\right\|=\left\|u_{64}^{0}-u_{64}\right\|$ on grid $64 \times 64$.

From Figure 2, the accuracy of the initial error on the next grid of RCMG method is higher than EXCMG method. 


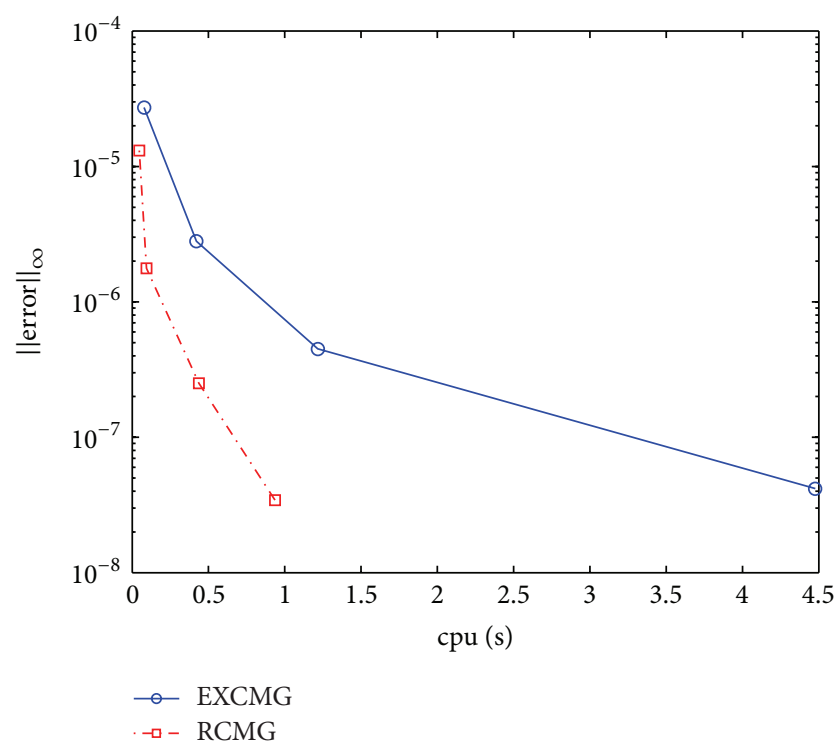

FIgURE 4: Comparison of the maximum error $\left\|\bar{u}^{L}-u\right\|_{\infty}$ and cpu time for Example 5 with $L=3$, taking step lengths $h_{L}=1 / m, m=$ $64,128,256$, and 512 , respectively.

TABLE 1: Numerical results of EXCMG and RCMG for Example 4.

\begin{tabular}{cccccc}
\hline$L$ & \multirow{3}{*}{$1 / h_{L}$} & $\left\|\bar{u}^{L}-u\right\|_{\infty}$ & $\mathrm{cpu}$ & $\left\|\bar{u}^{L}-u\right\|_{\infty}$ & $\mathrm{cpu}$ \\
\hline \multirow{3}{*}{3} & 128 & $6.28 E-07$ & 0.37 & $1.27 E-07$ & 0.14 \\
& 256 & $1.12 E-07$ & 1.12 & $1.85 E-08$ & 0.31 \\
& 512 & $1.07 E-08$ & 4.10 & $2.58 E-09$ & 1.12 \\
\hline \multirow{4}{*}{4} & 128 & $4.76 E-07$ & 0.34 & $2.61 E-07$ & 0.08 \\
& 256 & $1.27 E-07$ & 1.19 & $6.11 E-08$ & 0.42 \\
& 512 & $3.58 E-08$ & 4.38 & $6.07 E-09$ & 1.29 \\
\hline \multirow{3}{*}{5} & 128 & $9.21 E-07$ & 0.51 & $3.05 E-07$ & 0.08 \\
& 256 & $1.62 E-07$ & 1.06 & $7.82 E-08$ & 0.31 \\
& 512 & $2.72 E-08$ & 4.15 & $1.72 E-08$ & 1.15 \\
\hline
\end{tabular}

Namely, a better initial value on the fine grid can be got by using RCMG method. Based on the results of the literature [17-20], the RCMG method can obtain good convergence rate.

\subsection{Comparison between EXCMG Method and RCMG} Method. Let $\|$ Error $\left\|_{\infty}=\right\| \overline{\mathcal{u}}^{L}-u \|_{\infty}$ denote the maximum absolute error between the computed solution $\bar{u}^{L}$ and the exact solution $u$ on the finest grid points. The "cpu" denotes the computing time (unit: second) of EXCMG method and RCMG method.

From Figures 3 and 4 and Tables 1 and 2, we see that, under the same conditions, the RCMG method can obtain higher computational precision and spend less computing time than EXCMG method.
TABLE 2: Numerical results of EXCMG and RCMG for Example 5.

\begin{tabular}{cccccc}
\hline \multirow{2}{*}{$L$} & \multirow{3}{*}{$1 / h_{L}$} & $\left\|\bar{u}^{L}-u\right\|_{\infty}$ & $\mathrm{cpu}$ & $\left\|\bar{u}^{L}-u\right\|_{\infty}$ & $\mathrm{cpu}$ \\
\hline \multirow{3}{*}{3} & 128 & $2.80 E-06$ & 0.39 & $1.77 E-06$ & 0.16 \\
& 256 & $4.50 E-07$ & 1.22 & $2.50 E-07$ & 0.50 \\
& 512 & $4.18 E-08$ & 4.17 & $3.16 E-08$ & 1.23 \\
\hline \multirow{3}{*}{4} & 128 & $1.07 E-05$ & 0.25 & $4.29 E-06$ & 0.11 \\
& 256 & $1.92 E-06$ & 1.00 & $9.28 E-07$ & 0.30 \\
& 512 & $1.71 E-07$ & 4.23 & $1.39 E-07$ & 1.17 \\
\hline \multirow{3}{*}{5} & 128 & $1.74 E-05$ & 0.28 & $2.56 E-06$ & 0.09 \\
& 256 & $5.29 E-06$ & 1.01 & $2.00 E-06$ & 0.31 \\
& 512 & $7.94 E-07$ & 4.07 & $3.35 E-07$ & 1.06 \\
\hline
\end{tabular}

\section{Conclusion}

In this paper, based on a fourth order compact scheme, we present a Richardson cascadic multigrid method for $2 \mathrm{D}$ Poisson problem by using Richardson technique presented by [21]. The numerical results show that RCMG method has higher computational accuracy and higher efficiency.

\section{Conflict of Interests}

The authors declare that there is no conflict of interests regarding the publication of this paper.

\section{Acknowledgments}

This work is supported by the National Natural Science Foundation of China (Grant no. 11161014), the National Natural Science Foundation of Yunnan Province (Grant no. 2012FD054), and Scientific Research Starting Foundation for Master or Ph.D. of Honghe University (Grant no. XJ1S0925).

\section{References}

[1] R. E. Alcouffe, A. Brandt, J. E. Dendy et al., "The multigrid method or the diffusion equation with strongly discontinuous coefficients," SIAM Journal on Scientific and Statistical Computing, no. 2, pp. 430-454, 1981.

[2] C. E. Kees, C. T. Miller, E. W. Jenkins, and C. T. Kelley, "Versatile two-level Schwarz preconditioners for multiphase flow," Computational Geosciences, vol. 7, no. 2, pp. 91-114, 2003.

[3] C. Vuik, A. Segal, and J. A. Meijerink, "An efficient preconditioned CG method for the solution of a class of layered problems with extreme contrasts in the coefficients," Journal of Computational Physics, vol. 152, no. 1, pp. 385-403, 1999.

[4] B. Heise and M. Kuhn, "Parallel solvers for linear and nonlinear exterior magnetic field problems based upon coupled FE/BE formulations," Computing, vol. 56, no. 3, pp. 237-258, 1996.

[5] R. K. Coomer and I. G. Graham, "Massively parallel methods for semiconductor device modelling," Computing, vol. 56, no. 1, pp. 1-27, 1996.

[6] V. E. Howle and S. A. Vavasis, "An iterative method for solving complex-symmetric systems arising in electrical power modeling," SIAM Journal on Matrix Analysis and Applications, vol. 26, no. 4, pp. 1150-1178, 2005. 
[7] F. A. Bornemann and P. Deuflhard, "The cascadic multigrid method for elliptic problems," Numerische Mathematik, vol. 75, no. 2, pp. 135-152, 1996.

[8] Z.-C. Shi, X.-J. Xu, and Y.-Q. Huang, "Economical cascadic multigrid method (ECMG)," Science in China A, vol. 50, no. 12, pp. 1765-1780, 2007.

[9] Z.-C. Shi and X. Xu, "Cascadic multigrid method for elliptic problems," East-West Journal of Numerical Mathematics, vol. 7, no. 3, pp. 199-209, 1999.

[10] Z.-C. Shi and X.-J. Xu, "Cascadic multigrid for parabolic problems," Journal of Computational Mathematics, vol. 18, no. 5, pp. 551-560, 2000.

[11] Z. Shi and X. Xu, "A new cascadic multigrid," Science in China A, vol. 44, no. 1, pp. 21-30, 2001.

[12] V. V. Shaidurov, "Some estimates of the rate of convergence for the cascadic conjugate-gradient method," Computers and Mathematics with Applications, vol. 31, no. 4-5, pp. 161-171, 1996.

[13] R. Stevenson, "Nonconforming finite elements and the cascadic multi-grid method," Numerische Mathematik, vol. 91, no. 2, pp. 351-387, 2002.

[14] Y. Huang, Z. Si, T. Tang, and W. Xue, "A multilevel successive iteration method for nonlinear elliptic problems," Mathematics of Computation, vol. 73, no. 246, pp. 525-539, 2004.

[15] G. Timmermann, "A cascadic multigrid algorithm for semilinear elliptic problems," Numerische Mathematik, vol. 86, no. 4, pp. 717-731, 2000.

[16] D. Braess and W. Dahmen, "A cascadic multigrid algorithm for the Stokes equations," Numerische Mathematik, vol. 82, no. 2, pp. 179-191, 1999.

[17] C. Chen, Z.-C. Shi, and H. Hu, "On extrapolation cascadic multigrid method," Journal of Computational Mathematics, vol. 29, no. 6, pp. 684-697, 2011.

[18] C. M. Chen, Z. Q. Xie, C. L. Li et al., "Study of a new extrapolation multigrid method," Journal of Natural Science of Hunan Normal University, vol. 30, no. 2, pp. 1-5, 2007.

[19] C. M. Chen, H. L. Hu, Z. Q. Xie, and C. Li, "Analysis of extrapolation cascadic multigrid method (EXCMG)," Science in China A, vol. 51, no. 8, pp. 1349-1360, 2008.

[20] C. M. Chen, H. L. Hu, Z. Q. Xie et al., " $L^{2}$-error of extrapolation cascadic multigrid (EXCMG)," Acta Mathematica Scientia, vol. 29, no. 3, pp. 539-551, 2009.

[21] Y. Wang and J. Zhang, "Sixth order compact scheme combined with multigrid method and extrapolation technique for $2 \mathrm{D}$ poisson equation," Journal of Computational Physics, vol. 228, no. 1, pp. 137-146, 2009.

[22] J. Zhang, "Multigrid method and fourth-order compact scheme for 2D Poisson equation with unequal mesh-size discretization," Journal of Computational Physics, vol. 179, no. 1, pp. 170-179, 2002. 


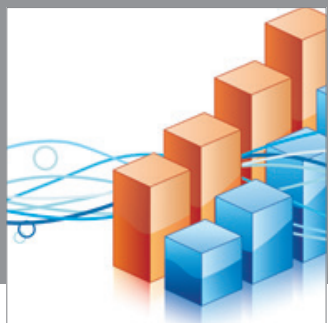

Advances in

Operations Research

mansans

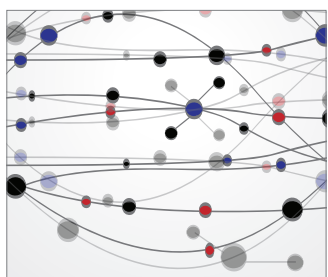

The Scientific World Journal
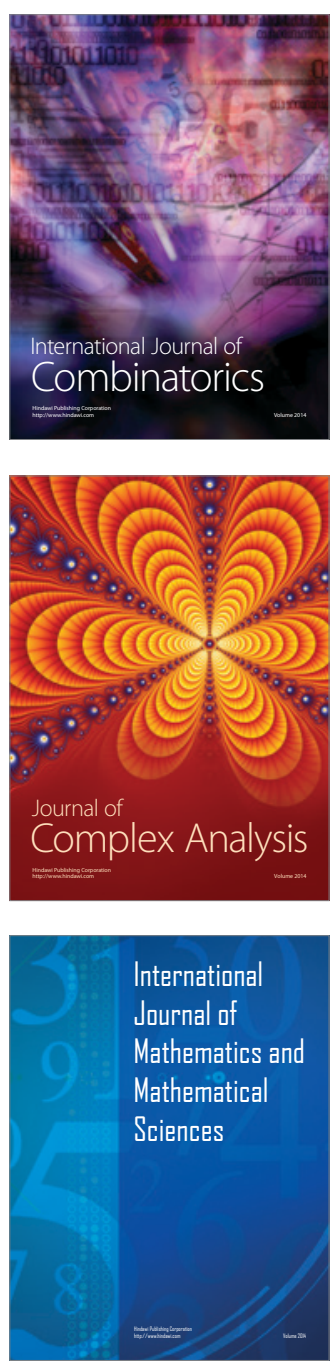
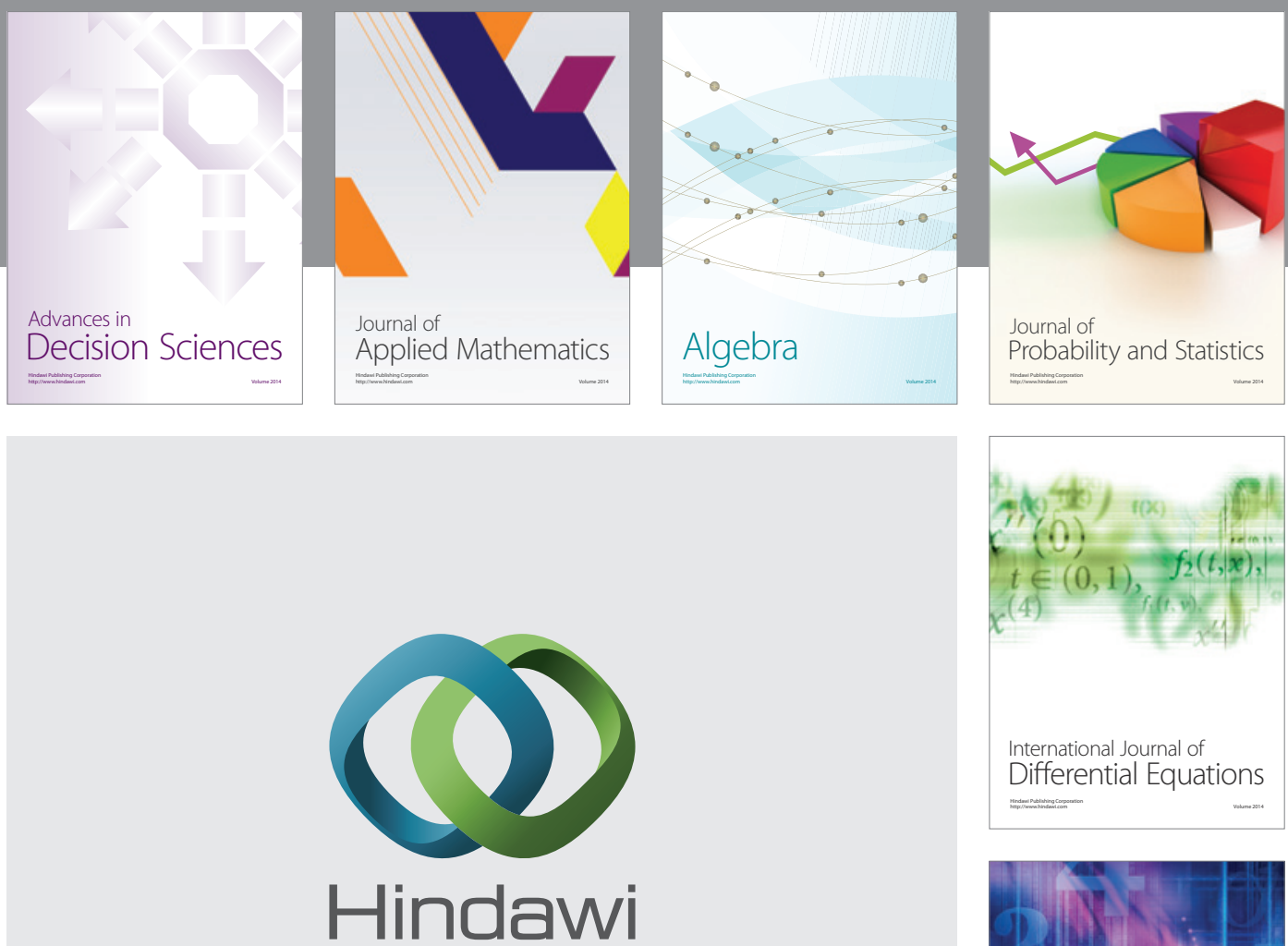

Submit your manuscripts at http://www.hindawi.com
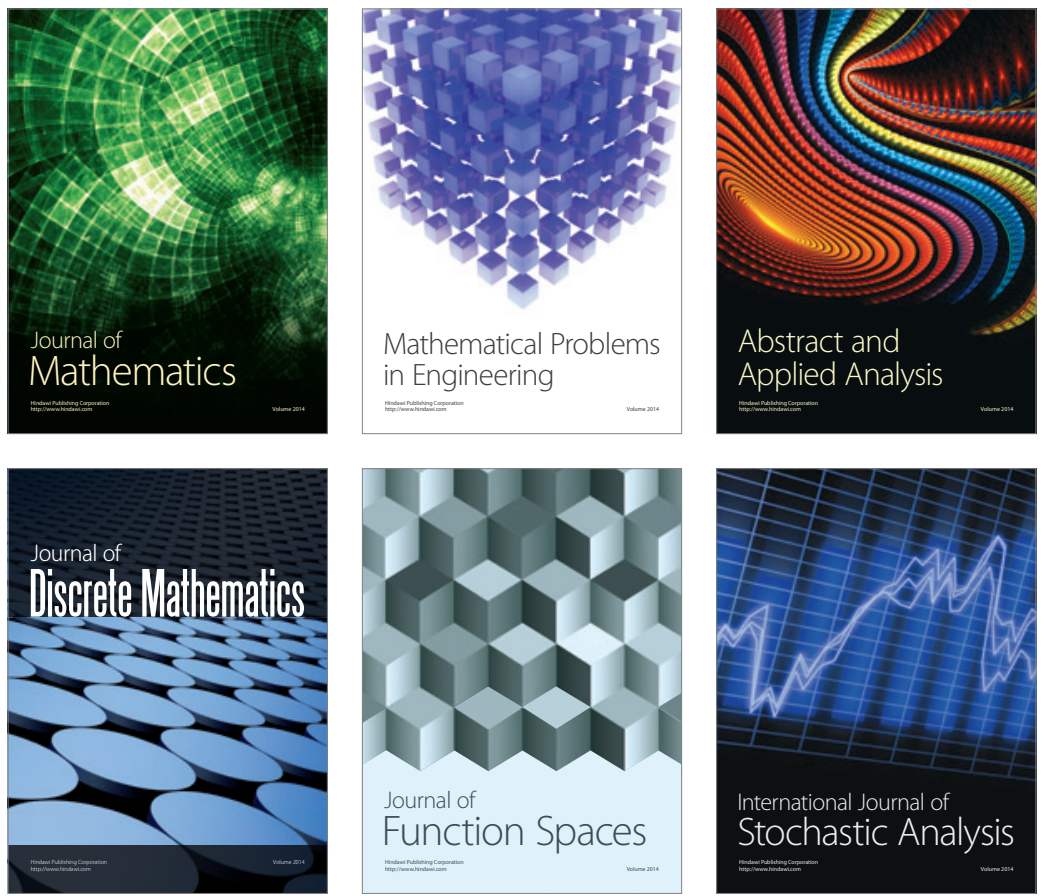

Journal of

Function Spaces

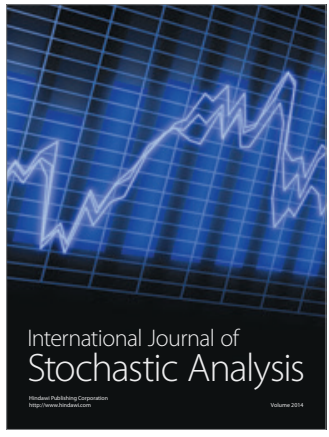

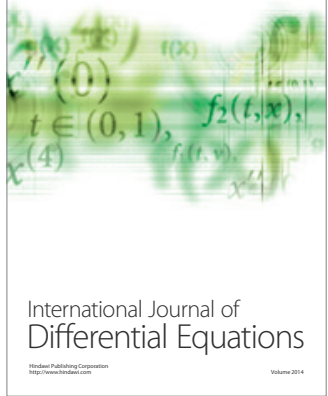
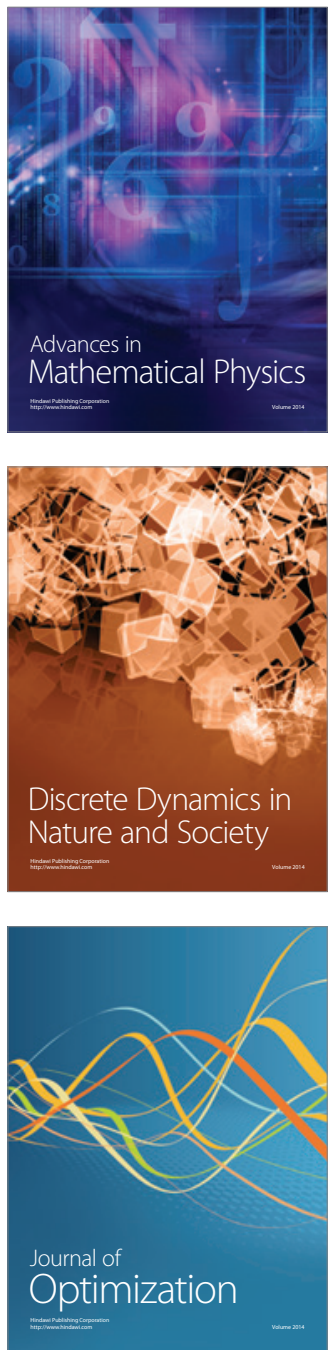